\title{
Specification, Modelling and Visualization of Arbitrarily Shaped Cut Surfaces in the Volume Model
}

\author{
B. Pflesser and U. Tiede and K.H. Höhne \\ Institute of Mathematics and Computer Science in Medicine (IMDM) \\ University-Hospital Eppendorf \\ Martinistraße 52, 20246 Hamburg, Germany \\ pflesser@uke.uni-hamburg.de
}

\begin{abstract}
So far, exploration of volume models is limited to cut planes or addition/removal of segmented objects. More capable exploration techniques are needed in order to allow a 'look and feel' close to a real dissection. This is especially important for applications like the simulation of osteotomy surgery. Therefore, we have developed methods for free-form volume-sculpting operations which allow the interactive specification, representation and high-quality rendering of free form regions. The novelty of this approach is that these regions are represented within the generalized-voxel-model, together with a simulation of the partialvolume-effect, which allows a sub-voxel localization of cut surfaces. These techniques are implemented in our VOXEL-MAN visualization system, thus enhancing the exploration techniques for volume data. Furthermore, we developed an extended ray-casting algorithm for 3D-visualization of object motion with detection and visualization of interpenetrating volumes. These methods together provide a powerful tool for volume exploration and applications like the rehearsal of surgical interventions.
\end{abstract}

\section{Introduction}

Systems for rehearsal and planning of surgical interventions are mostly based on traditional computer graphics methods where an object is represented by its surface only $[4,5,9]$. Often, these approaches concentrate on deformation techniques rather than cutting operations. For the specification of cuts, it is necessary to generate a new polygonal representation of cut regions. Therefore interactive specification tools are limited to basic techniques like cut planes and can not provide the 'look and feel' close to a real dissection. Furthermore, these approaches suffer from the lack of information about the interior of an object.

Several applications for object manipulation have been suggested that are based on the binary-voxel-model [16], which certainly does not overcome the limitation of surface based methods. The specification of arbitrarily shaped objects within the gray-level-volume model is a less developed subject. Some approaches have been presented by $[1,17]$. However, these applications do not provide flexible dissection tools or proper visualization methods for cut surfaces. In a first approach [7], we have developed a method for free form cutting based on gray level 
modification. This method provided a fairly good visualization of cut surfaces but proved to be not flexible enough for complex interactive cutting operations.

Therefore, we wanted to establish free form operations in the VOXEL-MAN volume visualization system $[11,13]$ which already provides a set of tools for object manipulation in a volume representation. For example, objects defined in a previous segmentation step may be removed or added like in an assembly kit, or arbitrary cut planes may be specified.

In order to enhance the potential of these techniques, the following qualitites are required for methods of specification, representation and visualization of free-form cut regions:

- Representation of cut regions with preservation of object information.

- Sub-voxel modelling of cut surfaces (position, shape and surface normals)

- Interactive and flexible cutting tools.

- High-quality visualization of cut regions with preservation of object surfaces.

\section{Method}

\subsection{Modelling of Cut Regions}

Attribute Level. Within the VOXEL-MAN system anatomical objects are described using a two level data structure [3]. The lower level is a discrete data volume, as obtained from a medical imaging system. In addition, a set of attributes is assigned to every voxel, indicating its membership to anatomical regions under various aspects (e.g. morphology, functional anatomy). This level is equivalent to the previously described generalized voxel model [2]. On the upper level, objects and their relations are described symbolically [8].

For achieving the new functionality of representing arbitrarily formed cutout regions within the generalized voxel model, we are using an additional voxel attribute. In contrast to other attributes, this kind of information is not static but subject to changes during the interactive specification process. By computing a table of all possible occurences of attribute combinations, it is possible to represent cut out regions without an additional label volume, thus minimizing memory requirements. This way, the original object information is available at any point of a cut-out region, and all operations can easily be reversed.

Data Level. Apparently, voxel attributes are limited to the resolution of the underlying data volume and do not provide means for a proper visualization of cut surfaces. An accurate estimation of surface normals is needed to visualize $3 \mathrm{D}$-objects. For rendering surfaces in tomographic volume data the gray-levelgradient method has proven to be accurate. Therefore, we model cut surfaces within a data volume where the partial-volume-effect, which is the prerequisite for the gray-level-gradient method, is calculated as it would be generated by an imaging system. This way, the gray-level-gradient method which provides an accurate determination of the surface shape can be applied for visualization of 
arbitrarily shaped cut surfaces. Furthermore, the determination of the position of a surface point can be achieved within sub-voxel resolution, so that the decision of whether an object has been cut or not is very accurate.

\subsection{Specification}

Interactive Tools. The specification of $3 \mathrm{D}$-areas within a data volume in the context of perspective 2D-images is not achieved easily. By utilizing the known image transformation an interactive tool can be moved arbitrarily in 3D-space (position and direction) and can be projected to image-space. The tip of such a tool can be defined in many ways, allowing the imitation of different instruments like a scalpel or a laser beam:

- Shape:

- Geometrical representations, e.g. cuboid, ellipsoid, cylinder.

- Polygonal models.

- Size and direction (relatively to direction of tool).

- "Sensibility": Which objects can be affected by an instrument?

- "Roughness": The modelling of the cut surface can be parameterized.

This tool kit provides very flexible methods for the specification of free-form regions within the volume model:

- Larger regions for the "in depth" exploration of the model.

- Gradual cutting with smaller instruments ("scalpel").

- Section anatomical parts.

Modelling of Cut Surfaces. Once shape and position of a tool have been specified, the voxel inside the tool can easily be determined and therefore be labeled. For an accurate representation of the tool shape in the data volume, it is necessary to estimate the partial-volume-effects on the surface very accurately. This can be achieved by calculating the percentage of a given sample region, which is covered by the tool tip. Since the tool tip is geometrically or polygonally represented, it can easily be determined whether a sample point lies inside the tool or not (Fig. 1 , left). Whenever this sampling process results in a gray value which satifies the threshold definition of the cut region, the voxel has to be labeled.

Especially for gradual cutting with a "scalpel"-like tool, it is important to preserve existing cut surfaces. Therefore, it is not sufficient to resample only the tool. Also the amount of an existing cut region which is unaffected by the new cutting has to be determined (Fig. 1, right). Since the geometry and position of the tool which generated these cut surfaces are no longer known, the region has to be resampled by gray-level interpolation. The decision of whether a sample point is inside the cut region or not, is then made by the threshold definition specified for the cut region. 

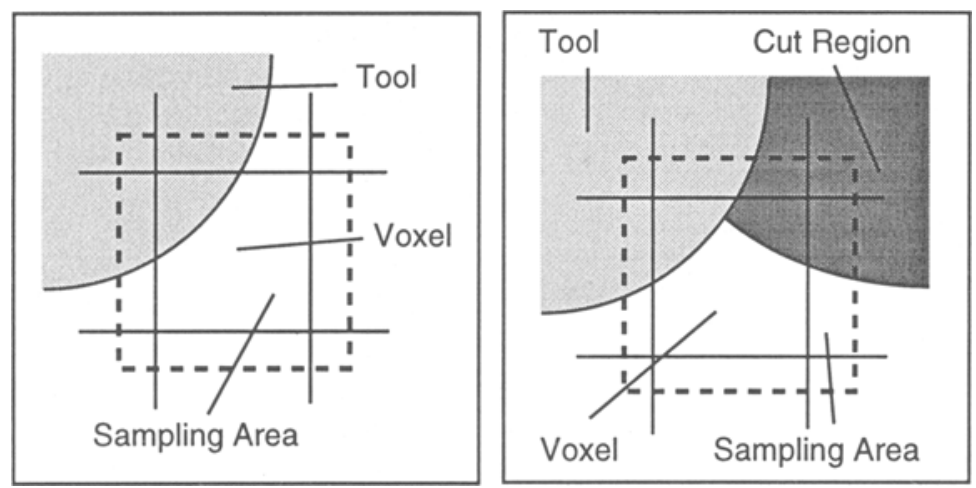

Fig. 1. Resampling of tool geometry for partial-volume-effect estimation (left). Resampling with preservation of existing cut surfaces (right).

\subsection{Visualization}

For rendering surfaces in tomographic volume data the gray-level-gradient method [14] has proven to be accurate. However, when visualizing a surface where a cut region has been removed, this method can not be used directly, because graylevel differences inside an object are not related to a surface. On the other hand the cut region itself is represented as an "artificial" object and the visualization of its surface would e.g. lack the morphological information. Therefore we extended the visualization algorithm in such a way that the morphological information and the information about cut surfaces are combined. Obviously it is not sufficient to make this combination with label information only. Rather, for each sample point during the ray casting process, the surfaces of morphological objects as well as cut surfaces have to be determined at subvoxel resolution. Both the morphological objects and cut-out regions are represented in the generalizedvoxel-model with partial-volume-effects. Hence the position of the surfaces can be determined precisely with gray-level-interpolation using thresholding, and the surface normal estimation at this position can be calculated using the gray-levelgradient method [15].

The key point here is to detect if a cut surface really truncates an object or if the object has not been affected by a cutting operation. The situation is clear when only a cut surface has been detected. In this case the object should be visualized using the cut surface information. When multiple surfaces have been found between successive sample points we must determine which surface should be used.

The situation of multiple surfaces can occur when:

- an object has been cut close to its surface

- an object has been uncovered by a selective cutting operation

In both cases the information about the location of the surfaces is not suffcient to determine which surface should be visualized. Therefore we developed 
a method of adaptive sampling: Fig. 2, left, shows an example of this situation. At the sample position $s 2$ an object which has been truncated by a cut region has been found. Between the successive sample points $s 1$ and $s 2$ we calculate the surface boundaries of the object and the cut region. Without the information which object is located between these surfaces the decision which surface (or any surface at all) should be visualized can not be made. Therefore we generate an additional sample point $(s 3)$ between the surface boundaries. In this case, the object is located at $s 3$, so it is visualized using its "original" surface.

Fig. 2, right, shows the situation where an object has been uncovered partly by a selective cutting operation. Clearly this part of the object should be visualized with its "original" surface. This situation can not be solved by adaptive sampling only because the cut region produces an "artificial" surface which should not be used for visualization. Hence the information about the objects to which a cutting operation is restricted is used during the ray casting process and a cut surface which affects a "forbidden" object is ignored.
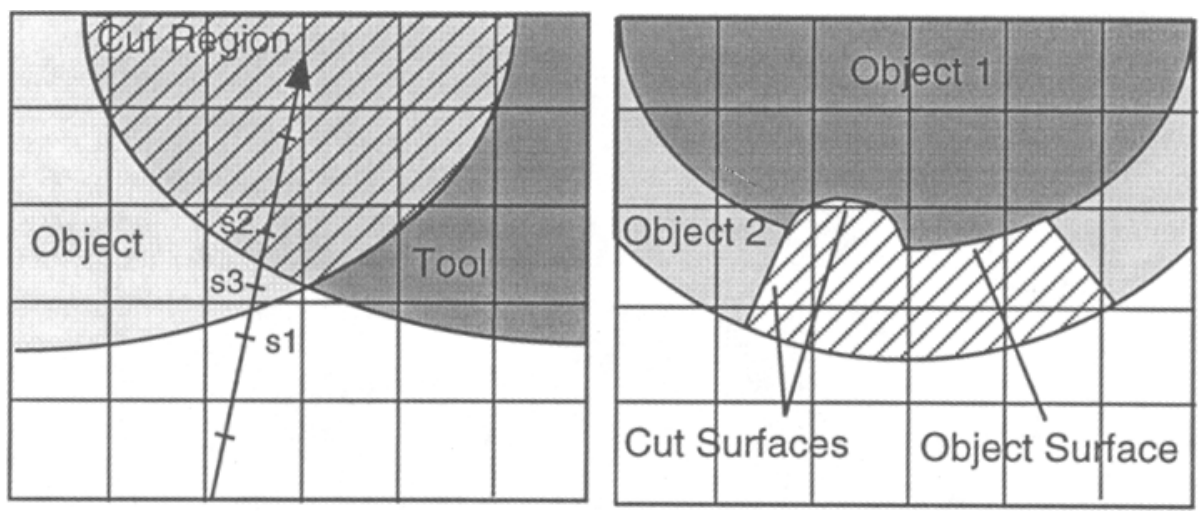

Fig. 2. Situation with multiple surfaces.

Left: an object has been cut close to its surface. With the method of adaptive sampling (sample point $s 3$ between the surfaces) it can be decided that the object is to be visualized with its "original" surface. Right: an object has been uncovered partly by a selective cutting operation. Since the cut operation has been restricted to object 2 the "artifical" cut surface close to object 1 is ignored and the object surface is used for visualization.

For applications like the simulation of osteotomy surgery the visualization of object motion is needed. Therefore we developed an extension of our ray casting algorithm (ray compositing) which allows the movement of objects in the volume model. A detailed description of this algorithm can be found in [6]. 


\section{Results}

Figure 3 demonstrates some of the capabilities of the described method. In the upper left image parts of soft tissue and bone have been resected and an interactively specified ellipsoid-shaped region has been removed. It can be seen that the surface normal estimation on the "artifical" cut surfaces is very accurate. The upper right image shows the simulation of an osteotomy. The blue lines indicate the planning of the osteotomy and show the principle of the "scalpel"-like specification method. A part of the frontal bone (a region which is limited by a closed contour) has been resected and moved forward.

Since modelling and representation of cut surfaces is independent from the representation of the anatomical objects, our method can be applied identically to the data of the Visible Human Project ${ }^{T M}$ [12]. Figure 3, lower left, shows how the anatomy of the visual system can be explored within the context of surrounding structures by specifying a cut region where the eyes, optic tracts and optic muscles have been excluded from the operation. The lower right image demonstrates the simulation of an anatomical dissection. The abdomen have been opened and parts of the large and small bowels have been removed in order to uncover the right kidney.

\section{Conclusion}

The novel approach presented in this paper provides powerful exploration techniques as well as the basis for simulation of surgical interventions in the voxelmodel which could not be achieved with any surface-based method. Arbitrarily shaped cut regions are represented in the volume model with proper modelling of the surface normals via simulating the partial-volume-effect. This method allows the sub-voxel localization of cut surfaces and the accurate calculation of surface normals using the gray-level-gradient method. This way, a realistic rendering which greatly facilitates spatial perception can be achieved. Since the representation of a cut surface is iteratively modified, arbitrarily complex surfaces can be modelled, which can not be achieved by surface based representations. We have implemented a wide range of flexible and interactive specification tools, as needed for surgical simulation. In combination with the technique for simulation of object movement, these methods create a capable tool kit for applications in the field of planning and rehearsal of surgical interventions.

For the future, one exciting field will be the combination of this approach with methods for elastic object deformation, also implemented in the VOXEL-MAN environment [10]. This way, applications may be extended to a more realistic handling of soft tissue as well.

\section{References}

1. Arridge, S. R.: Manipulation of Volume Data for Surgical Simulation. In Höhne, K. H. et al. (Eds.): 3D-Imaging in Medicine: Algorithms, Systems, Applications, NATO ASI Series F 60, Springer-Verlag, Berlin, 1990, 289-300. 

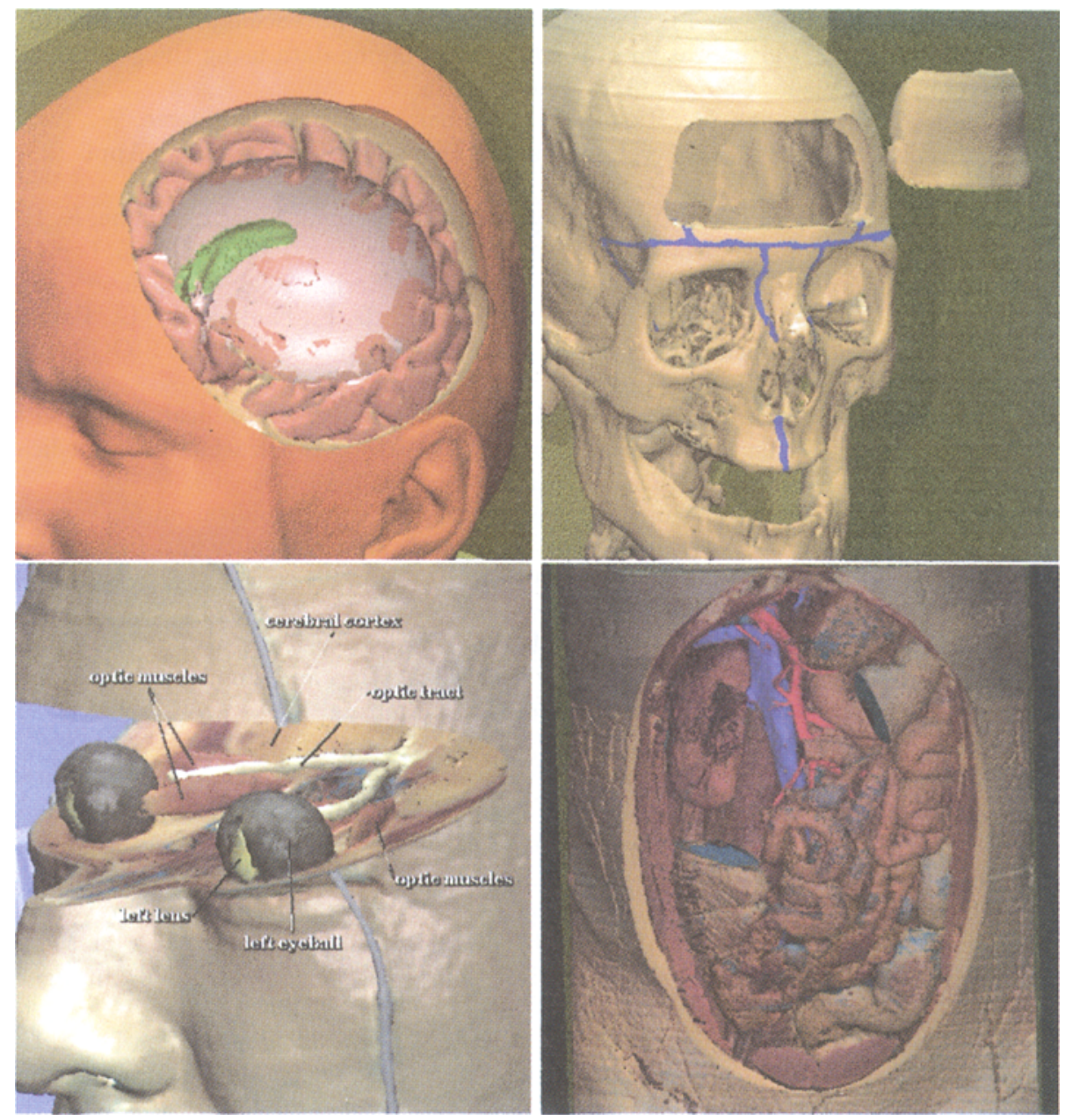

Fig. 3. Visualization of Cut Surfaces:

Upper left: Parts of soft tissue and bone have been resected and the model have been further explored "in depth" by removal of an ellipsoid shape.

Upper right: Simulation of craniofacial surgery. The blue lines represent the planning of the operation and a part of the frontal bone has been removed using a "scalpel".

Lower left: The extended exploration possibilities are demonstrated with data from the Visible Human Project. The eyes, optic tract and optic muscles have been uncovered by removal of an ellipsoidal region.

Lower right: This image demonstrates the simulation of an anatomical dissection. The abdomen have been opened and parts of the large and small bowel have been removed in order to uncover the right kidney.

Note: The smooth appearance of the cut surfaces is achieved by our new method which simulates the partial-volume-effect. 
2. Höhne, K. H., Bomans, M., Pommert, A., Riemer, M., Schiers, C., Tiede, U., Wiebecke, G.: 3D-visualization of tomographic volume data using the generalized voxel-model. Visual Comput. 6, 1 (1990), 28-36.

3. Höhne, K. H., Bomans, M., Riemer, M., Schubert, R., Tiede, U., Lierse, W.: A 3D anatomical atlas based on a volume model. IEEE Comput. Graphics Appl. 12, 4 (1992), 72-78.

4. Keeve, E., Girod, S., Girod, B.: Craniofacial Surgery Simulation. In Höhne, K. H., Kikinis, R. (Eds.): Visualization in Biomedical Computing, Proc. VBC '96, Lecture Notes in Computer Science 1131, Springer-Verlag, Berlin, 1996, 541-546.

5. Koch, R. M., Gross, M. H., Carls, F. R., von Büren, D. F., Fankhauser, G., Parish, Y. I. H.: Simulating facial surgery using finite element models. In Proc. SIGGRAPH 96. New Orleans, LA, 1996, 421-428.

6. Pflesser, B., Tiede, U., Höhne, K. H.: Simulating motion of anatomical objects with volume-based 3D-visualization. In Robb, R. A. (Ed.): Visualization in Biomedical Computing 1994, Proc. SPIE 2359. Rochester, MN, 1994, 291-300.

7. Pflesser, B., Tiede, U., Höhne, K. H.: Towards realistic visualization for surgery rehearsal. In Ayache, N. (Ed.): Computer Vision, Virtual Reality and Robotics in Medicine, Proc. CVRMed '95, Lecture Notes in Computer Science 905, SpringerVerlag, Berlin, 1995, 487-491.

8. Pommert, A., Schubert, R., Riemer, M., Schiemann, T., Tiede, U., Höhne, K. H.: Symbolic modeling of human anatomy for visualization and simulation. In Robb, R. A. (Ed.): Visualization in Biomedical Computing 1994, Proc. SPIE 2359. Rochester, MN, 1994, 412-423.

9. Reinig, K., Spitzer, V., Pelster, H., Johnson, T., Mahalik, T.: More Real-Time Visual and Haptic Interaction with Anatomical Data. In Morgan, K. et al. (Eds.): Medicine Meets Virtual Reality: Global Healthcare Grid, MMVR'97, Studies in Health Technology and Informatics 39, IOS Press, Amsterdam, 1997, 155-158.

10. Schiemann, T., Höhne, K. H.: Definition of volume transformations for volume interaction. In Duncan, J., Gindi, G. (Eds.): Information Processing in Medical Imaging, Proc. IPMI '97, Lecture Notes in Computer Science 1230, SpringerVerlag, Berlin, 1997, 245-258.

11. Schubert, R., Höhne, K. H., Pommert, A., Riemer, M., Schiemann, T., Tiede, U., Lierse, W.: A new method for practicing exploration, dissection, and simulation with a complete computerized three-dimensional model of the brain and skull. Acta Anat. 150, 1 (1994), 69-74.

12. Spitzer, V., Ackerman, M. J., Scherzinger, A. L., Whitlock, D.: The Visible Human male: A technical report. J. Am. Med. Inf. Ass. 3, 2 (1996), 118-130.

13. Tiede, U., Bomans, M., Höhne, K. H., Pommert, A., Riemer, M., Schiemann, T., Schubert, R., Lierse, W.: A computerized three-dimensional atlas of the human skull and brain. Am. J. Neuroradiology 14, 3 (1993), 551-559.

14. Tiede, U., Höhne, K. H., Bomans, M., Pommert, A., Riemer, M., Wiebecke, G.: Investigation of medical 3D-rendering algorithms. IEEE Comput. Graphics Appl. 10, 2 (1990), 41-53.

15. Tiede, U., Schiemann, T., Höhne, K. H.: High quality rendering of attributed volume data. In Proc. IEEE Visualization '98, IEEE Computer Society Press, 1998. (accepted for publication).

16. Udupa, J. K., Odhner, D.: Fast visualization, manipulation and analysis of binary volumetric objects. IEEE Comput. Graphics Appl. 11, 6 (1991), 53-62.

17. Yasuda, T., Hashimoto, Y., Yokoi, S., Toriwaki, J.-I.: Computer system for craniofacial surgical planning based on CT images. IEEE Trans. Med. Imaging MI-9, 3 (1990), 270-280. 